\title{
Linear programs for resource sharing among heterogeneous agents: the effect of random agent arrivals
}

\author{
Alessandro Falsone, Kostas Margellos, Simone Garatti, Maria Prandini
}

\begin{abstract}
We consider a multi-agent resource sharing problem that can be represented by a linear program. The amount of resource to be shared is fixed, and each agent adds to the linear cost and constraint a term that depends on some randomly extracted parameters, thus modelling heterogeneity among agents. We study the probability that the arrival of a new agent does not affect the optimal value and the resource share of the other agents, which means that the system cannot accommodate the request of a further agent and has reached its saturation limit. In particular, we determine the maximum number of requests for the shared resource that the system can accommodate in a probabilistic sense. This result is proven by first formulating the dual of the resource sharing linear program, and then showing that this is a random linear program. Using results from the scenario theory for randomized optimization, we bound the probability of constraint violation for the dual optimal solution, and prove that this is equivalent with the primal optimal value and resource share remaining unchanged upon arrival of a new agent. We discuss how this can be thought of as probabilistic sensitivity analysis and offer an interpretation of this setting in an electric vehicle charging control problem.
\end{abstract}

\section{INTRODUCTION}

Systems with multiple agents interacting with each other sharing common resources appear in many engineering disciplines. Power networks [1], [2], [3], demand side management [4], [5], social networks [6], [7], [8], consensus and flocking [9], [10], robotic and sensor networks [11], [12], constitute only some multi-agent application domains of contemporary research interest.

According to the particular application, and the behaviour governing the agents' interaction, two cases can be distinguished: a cooperative set-up, where agents aim to share common resources in view of achieving a social welfare optimum; and a non-cooperative set-up, where agents act as self-interested entities, competing against shared resources. In the former case, research has been mainly concentrated towards the development of distributed optimization regimes based on iterative algorithms (see [13] and references therein, and [14], [15], [16], [17] for recent contributions), that enable parallelizing computation with agents solving at each iteration only a local problem without exchanging with the other agents information that is considered private (e.g., utility and

This work is partially supported by the European Commission under the project UnCoVerCPS with grant number 643921.

Alessandro Falsone, Simone Garatti and Maria Prandini are with Dipartimento di Elettronica, Informazione e Bioingegneria, Politecnico di Milano, Piazza Leonardo da Vinci 32, 20133 Milano, Italy, e-mail: \{alessandro.falsone, simone.garatti, maria.prandini\}@polimi.it

Kostas Margellos is with the Department of Engineering Science, University of Oxford, Parks Road, Oxford, OX1 3PJ, United Kingdom, e-mail: kostas.margellos@eng.ox.ac.uk constraint functions). In the latter case where agents act in a non-cooperative manner, a multi-agent gaming set-up is typically adopted. Main research objective in this direction has been the characterization and distributed computation of Nash equilibrium strategies for the associated game [18], [19], [20].

Despite the intense research activity in control and optimization for multi-agent systems, most of the effort has been devoted towards the development of distributed algorithms for social welfare optimum or Nash equilibrium computation. To the best of our knowledge, there have only been just a few attempts in quantifying the capacity of the system (either in a cooperative or a non-cooperative setting) in terms of the maximum number of agents that can be accommodated so that, upon arrival of a new agent, the solutions of the others remain unaltered; see [4] for such an attempt in demand side management. Motivated by such concerns, in this paper we aim at addressing this problem, offering theoretical support for the developments in [4].

We consider a multi-agent resource sharing problem that can be represented by a linear program subject to budget constraints. We assume that the amount of resource to be shared is fixed, and each agent adds to the linear cost and constraint terms that are random, drawn from some fixed but possibly unknown probability distribution, thus modelling heterogeneity among agents. We study the probability that the arrival of a new agent does not affect the optimal value and the resource share of the other agents. As a result, the system has reached saturation, and can not accommodate the request of a further agent. In particular, we determine the maximum number of requests for the shared resource that the system can accommodate in a probabilistic sense. An alternative interpretation is that, after reaching a certain capacity limit, there is no incentive for a new agent to join a multi-agent resource sharing scheme.

This is proven by first formulating the dual of the resource sharing linear program, and then showing that this is a random linear program. Using results from the scenario approach theory for randomized optimization [21], [22], [23], we bound the probability of constraint violation for the dual optimal solution, and prove that this is equivalent to the primal optimal value and resource share remaining unchanged upon arrival of a new agent. We discuss how this can be thought of as probabilistic sensitivity analysis and offer an interpretation of this setting in an electric vehicle charging control problem.

The remainder of the paper is structured as follows. Section II states the resource sharing program under study and introduces the problem of identifying the maximum 
agent capacity, in a probabilistic sense. In Section III we provide some introductory results based on linear programming theory, whereas the main result of maximum agent capacity is proven in Section IV. Finally, Section V presents some numerical simulations, while Section VI concludes the paper and provides directions for future work.

\section{Problem Statement}

Consider the following multi-agent resource sharing problem with $m$ agents:

$$
\begin{aligned}
\mathcal{P}_{m}: \min _{\left\{x_{i} \in \mathbb{R}^{n_{i}}\right\}_{i=1}^{m}} & \sum_{i=1}^{m} c_{i}^{\top} x_{i} \\
\text { subject to: } & \sum_{i=1}^{m} A_{i} x_{i}=b \\
& x_{1}, \ldots, x_{m} \geq 0,
\end{aligned}
$$

where the vector $b \in \mathbb{R}^{p}$ is given and represents the total amount of an available resource that has to be shared among the $m$ agents. For all $i=1, \ldots, m, x_{i} \in \mathbb{R}^{n_{i}}$ denotes the vector of decision variables for agent $i, c_{i} \in \mathbb{R}^{n_{i}}$ and $A_{i} \in \mathbb{R}^{p \times n_{i}}$. Possibly, $n_{i} \neq n_{j}, i \neq j$. Problem $\mathcal{P}_{m}$ then involves $m$ agents trying to share the resource vector $b$, so as to minimize a global cost. All equalities and all inequalities are meant componentwise.

Each agent $i, i=1, \ldots, m$, is fully characterized by the tuple $\delta_{i}=\left(n_{i}, c_{i}, A_{i}\right)$. Here, we assume that, for each $i=1, \ldots, m, \delta_{i}$ denotes a sample/extraction of a random quantity $\delta=(\bar{n}, \bar{c}, \bar{A})$ defined over a probability space $(\Delta, \mathcal{D}, \mathbb{P})$, and all samples are extracted in an i.i.d. fashion. $\Delta$ denotes a generic set, not necessarily endowed with a metric, which, together with the $\sigma$-algebra $\mathcal{D}$ and the probability measure $\mathbb{P}$, forms a probability space. It should be noted that $\mathbb{P}$ corresponds to the joint probability distribution of the elements of $(\bar{n}, \bar{c}, \bar{A})$; in the particular case where all agents have decision vectors of the same length, then the marginal probability of $\bar{n}$ will be concentrated to that value. Since all samples are i.i.d., the collection $\left\{\delta_{i}\right\}_{i=1}^{m}$ is distributed according to the product measure $\mathbb{P}^{m}$.

Under this setting, $\mathcal{P}_{m}$ becomes a random linear program, with the number of agents serving as the number of realizations of the uncertain tuple $(\bar{n}, \bar{c}, \bar{A})$ that have instantiated $\mathcal{P}_{m}$. We further impose the following assumption on $\mathcal{P}_{m}$.

Assumption 1: For any $m \in \mathbb{N}$, the linear program $\mathcal{P}_{m}$ is feasible and admits a unique minimizer almost surely with respect to $\mathbb{P}^{m}$.

The uniqueness part of the assumption can be relaxed, by assuming that in case of multiple minimizers we single out a specific one by means of a deterministic tie-break rule.

Suppose that a new agent characterized by $\delta=(\bar{n}, \bar{c}, \bar{A})$ joins the resource sharing problem, and let $\bar{x} \in \mathbb{R}^{\bar{n}}$ denote its decision vector. The resulting $\mathcal{P}_{m+1}$ program accounting also for the new $\delta$ is denoted as $\mathcal{P}_{m, \delta}$ and is given by

$$
\begin{aligned}
& \mathcal{P}_{m, \delta}: \quad \min _{\left\{x_{i} \in \mathbb{R}^{n_{i}}\right\}_{i=1}^{m}, \bar{x} \in \mathbb{R}^{\bar{n}}} \sum_{i=1}^{m} c_{i}^{\top} x_{i}+\bar{c}^{\top} \bar{x} \\
& \text { subject to: } \quad \sum_{i=1}^{m} A_{i} x_{i}+\bar{A} \bar{x}=b \\
& x_{1}, \ldots, x_{m}, \bar{x} \geq 0 \text {. }
\end{aligned}
$$

Denote by $n=\sum_{i=1}^{m} n_{i}$ the total number of decision variables in $\mathcal{P}_{m}$, and let $x=\left[x_{1}^{\top} \ldots x_{m}^{\top}\right]^{\top} \in \mathbb{R}^{n}$. Similarly, $x_{\delta}=\left[x^{\top} \bar{x}^{\top}\right]^{\top} \in \mathbb{R}^{n+\bar{n}}$ is a vector containing the decision variables of $\mathcal{P}_{m, \delta}$. Let also $x^{\star}$ and $x_{\delta}^{\star}$ denote the optimal solutions of $\mathcal{P}_{m}$ and $\mathcal{P}_{m, \delta}$, respectively, which are considered to be unique based on Assumption 1 .

For a resource sharing problem with $m$ agents, our objective is to quantify how likely it is that the arrival of a new agent does not alter the optimal solution achieved by the initial $m$ agents alone. In other words, since agents are characterized by a stochastic tuple, we are interested in establishing a probabilistic statement of the form

$$
\mathbb{P}\left\{\delta=(\bar{n}, \bar{c}, \bar{A}) \in \Delta: x_{\delta}^{\star}=\left(x^{\star}, 0\right)\right\} \geq 1-\varepsilon,
$$

i.e., the probability that a new agent shows up such that its optimal decision vector $\bar{x}^{\star}$ is zero, and hence $x_{\delta}^{\star}=\left(x^{\star}, 0\right)$, is no less than a certain threshold $1-\varepsilon$. Note, however, that $x^{\star}$ is itself a random variable defined on the product space $\Delta^{m}$, since it depends on the samples $\left\{\delta_{i}\right\}_{i=1}^{m}$ (this dependency is not shown explicitly to simplify notation). Therefore, claim (3) can only be true with a certain confidence with respect to $\mathrm{P}^{m}$. The evaluation of this confidence for any given probability level $\varepsilon \in(0,1)$ and number of agents $m$ is the main result of this paper and is established in Section IV. To achieve this, Section III provides some theoretical machinery and intermediate results from linear programming.

\section{LINEAR PROGRAMMING RESULTS}

\section{A. Optimality condition}

Consider the random program $\mathcal{P}_{m}$ and let $A=$ $\left[A_{1} \cdots A_{m}\right] \in \mathbb{R}^{p \times n}$ and $c=\left[c_{1}^{\top} \ldots c_{m}^{\top}\right]^{\top} \in \mathbb{R}^{n}$. We are interested in the case where $n>p$, i.e., $\mathcal{P}_{m}$ has more decision variables than coupling constraints, as it is typically the case in resource sharing problems. Without loss of generality, we will henceforth assume that $A$ is full rowrank, i.e., all redundant constraints have been removed.

The equality constraint in $\mathcal{P}_{m}$, together with the nonnegativity constraints, defines almost surely a polyhedron, say $Q=\{x \geq 0: A x=b\} \subseteq \mathbb{R}^{n}$. Consider the vertices of $Q$. By Theorems 2.3-2.4 of [24], to any vertex $x^{v}$ there exists a set $\mathcal{J}=\left\{j_{1}, \ldots, j_{p}\right\} \subset\{1, \ldots, n\}$ of indices with $|\mathcal{J}|=p$ (i.e., its cardinality equals the number of rows of A) such that:

i) the columns $A_{j}, j \in \mathcal{J}$, of $A$, are linearly independent; ii) $x_{j}^{v}=0$ for all $j \notin \mathcal{J}$, where $x_{j}^{v}$ is the $j$-th component of $x^{v}$.

Thus, any vertex $x^{v}$ determines a partition of itself into two sub-vectors $x_{B}^{v}$ and $x_{N}^{v}$, where $x_{B}^{v}$ is a stacked vector containing the decision variables of agents with indices in 
$\mathcal{J}$, while $x_{N}^{v}$ those with indices in the complement of $\mathcal{J}$. The elements of $x_{B}^{v}$ and $x_{N}^{v}$ are referred to as basic and non-basic variables, respectively.

Corresponding to the partition of a vertex $x^{v}$ in basic and non-basic variables, denote by $A_{B}=\left[A_{j_{1}} \cdots A_{j_{p}}\right]$ the matrix obtained by the columns of $A$ corresponding to the indices in $\mathcal{J}$ and by $A_{N}$ the matrix constructed by removing from $A$ the columns with indices in $\mathcal{J}$. Since $x^{v}$ is feasible for $\mathcal{P}_{m}$, we have that

$$
A x^{v}=A_{B} x_{B}^{v}+A_{N} x_{N}^{v}=b .
$$

Due to the fact that $A_{B}$ has linearly independent columns by i) and $x_{N}^{v}=0$ by ii), we have that $x_{B}^{v}=A_{B}^{-1} b$.

Under Assumption 1, almost surely with respect to $\mathbb{P}^{m}$, it is well known that the optimal solution $x^{\star}$ of $\mathcal{P}_{m}$ occurs at a vertex of $Q$; see e.g. Theorem 2.7 of [24]. We then have the following proposition whose proof follows from [24] and the distinction between basic and non-basic variables presented above; we present it here for completeness. To this purpose, let $c_{B}$ and $c_{N}$ be a partition of $c$ corresponding to the partition of some vertex $x^{v}$ in basic and non-basic variables.

Proposition 1: Under Assumption 1, for any $m \in \mathbb{N}$ and almost surely with respect to $\mathbb{P}^{m}$, we have that

$$
c_{N}^{\top}-c_{B}^{\top} A_{B}^{-1} A_{N} \geq 0,
$$

if and only if $x^{v}$ is the optimal solution $x^{\star}$ of $\mathcal{P}_{m}$.

Proof: Under Assumption 1, $x^{\star}$ and $Q$ are well defined almost surely with respect to $\mathbb{P}^{m}$. For any given vertex $x^{v}$ of $Q$, consider a feasible point $x \in Q$, and let $z=x-x^{v}$. By feasibility of the solutions, $A x^{v}=b=A x$, and as a result, $A z=A\left(x-x^{v}\right)=0$, and hence $A_{B} z_{B}+A_{N} z_{N}=0$, where $z_{B}$ and $z_{N}$ is the partition of $z$ corresponding to the partition of $x^{v}$ in basic and nonbasic variables. We thus have that $z_{B}=-A_{B}^{-1} A_{N} z_{N}$. Letting $x_{B}$ and $x_{N}$ be the partition of $x$ corresponding to the partition of $x^{v}$, consider the cost function increment $c^{\top} z$ when moving from $x^{v}$ to $x$. We have that

$$
\begin{aligned}
c^{\top} z & =c_{B}^{\top} z_{B}+c_{N}^{\top} z_{N} \\
& =\left(c_{N}^{\top}-c_{B}^{\top} A_{B}^{-1} A_{N}\right) z_{N} \\
& =\left(c_{N}^{\top}-c_{B}^{\top} A_{B}^{-1} A_{N}\right)\left(x_{N}-x_{N}^{v}\right) \\
& =\left(c_{N}^{\top}-c_{B}^{\top} A_{B}^{-1} A_{N}\right) x_{N},
\end{aligned}
$$

where the second equality follows by substitution of $z_{B}=$ $-A_{B}^{-1} A_{N} z_{N}$, and the last one is due to the fact that $x_{N}^{v}=0$. Since $x_{N} \geq 0$ as a result of $x$ being feasible for $\mathcal{P}_{m},(5)$ is satisfied if and only if $c^{\top} z \geq 0$ for all admissible $z$. Since $c^{\top} z \geq 0$ for all admissible $z$ is equivalent to $c^{\top} x \geq c^{\top} x^{v}$ for all $x \in Q$, i.e., the optimality condition for $x^{v}$, we eventually have that (5) is satisfied if and only if $x^{v}$ is optimal for $\mathcal{P}_{m}$. This concludes the proof.

The quantity on the left-hand side of (5) is referred to as vector of "reduced costs". In the sequel, with a slight abuse of notation, we will denote by $A_{B}, A_{N}$ and $c_{B}, c_{N}$ the partition of $A$ and $c$ corresponding to the partition of the optimal solution $x^{\star}$ (and not of an arbitrary vertex in $Q$ ) into its basic and non-basic variables $x_{B}^{\star}$ and $x_{N}^{\star}$. Given this notation, note also that as a result of (4), by Proposition 1 we have that

$$
x_{B}^{\star}=A_{B}^{-1} b .
$$

Finally, it is perhaps worth mentioning that in case Assumption 1 is relaxed and the minimizer of $\mathcal{P}_{m}$ is not unique the aforementioned statements hold for some of the minimizers of $\mathcal{P}_{m}$.

\section{B. The dual problem}

Consider now the dual program corresponding to $\mathcal{P}_{m}$ (see Chapter 5 of [25]):

$$
\begin{aligned}
& \mathcal{D}_{m}: \max _{\lambda \in \mathbb{R}^{p}} \quad-\lambda^{\top} b \\
& \text { subject to: } \quad c_{i}^{\top}+\lambda^{\top} A_{i} \geq 0, \text { for all } i=1, \ldots, m \text {. }
\end{aligned}
$$

where $\lambda \in \mathbb{R}^{p}$ is the vector of dual variables, each of them corresponding to every row-wise coupling constraint in $\mathcal{P}_{m}$.

We then have the following proposition, which provides an expression for the optimal vector of dual variables.

Proposition 2: For any $m \in \mathbb{N}$, consider the dual program $\mathcal{D}_{m}$ corresponding to $\mathcal{P}_{m}$. Under Assumption 1,

$$
\lambda^{\star}=-\left(c_{B}^{\top} A_{B}^{-1}\right)^{\top},
$$

is an optimal solution of $\mathcal{D}_{m}$ almost surely with respect to $\mathbb{P}^{m}$.

Proof: We will first show that $\lambda^{\star}=-\left(c_{B}^{\top} A_{B}^{-1}\right)^{\top}$ is a feasible solution for $\mathcal{D}_{m}$. To this end, by Proposition 1, we have that $c_{N}^{\top}+\left(\lambda^{\star}\right)^{\top} A_{N}=c_{N}^{\top}-c_{B}^{\top} A_{B}^{-1} A_{N} \geq 0$, while $c_{B}^{\top}+\left(\lambda^{\star}\right)^{\top} A_{B}=c_{B}^{\top}-c_{B}^{\top} A_{B}^{-1} A_{B}=0$. Therefore,

$$
c_{i}^{\top}+\left(\lambda^{\star}\right)^{\top} A_{i} \geq 0, \text { for all } i=1, \ldots, m,
$$

implying that $\lambda^{\star}$ is feasible for $\mathcal{D}_{m}$. It remains to show that it is also optimal; to this end, consider the dual objective function evaluated at $\lambda^{\star}$. In particular,

$$
\begin{aligned}
-\left(\lambda^{\star}\right)^{\top} b & =c_{B}^{\top} A_{B}^{-1} b \\
& =c_{B}^{\top} x_{B}^{\star} \\
& =c_{B}^{\top} x_{B}^{\star}+c_{N}^{\top} x_{N}^{\star} \\
& =c^{\top} x^{\star},
\end{aligned}
$$

where the second equality is due to (7) and the third equality is due to the fact that $x_{N}^{\star}=0$. By weak duality [25], $-\lambda^{\top} b \leq$ $c^{\top} x^{\star}$ for any $\lambda$ feasible for (8). Equation (10) then implies that $\lambda^{\star}$ is optimal for $\mathcal{D}_{m}$, thus concluding the proof.

\section{ProbabilistiC MAXIMUM AGENT CAPACITY}

In this section we will use the scenario approach theory for random convex programs [21], [22], [23], to quantify the probability that the arrival of a new agent will not affect the optimal solution of $\mathcal{P}_{m}$. This theory was originally developed to accompany the primal solution of a random convex program with a probabilistic certificate regarding its feasibility properties. The random primal program $\mathcal{P}_{m}$, however, does not exhibit the structure required by the aforementioned references for the theory to be applicable. In this section, we will show that such a structure pertains the dual program; we will thus employ the scenario approach 
theory for $\mathcal{D}_{m}$, and by means of Propositions 1 and 2 , provide, with certain confidence, a statement of the form of (3).

\section{A. The scenario approach}

We revisit in this subsection the standard scenario approach theory by applying it to the linear program $\mathcal{D}_{m}$, i.e., the dual of $\mathcal{P}_{m}$. It should be noted that the scenario approach results hold for convex programs, and are not necessarily limited to linear programs; here we limit our presentation to linear programs due to our interest in $\mathcal{D}_{m}$ and $\mathcal{P}_{m}$.

Consider $\mathcal{D}_{m}$ and recall that $\lambda^{\star}$, as defined by (9), denotes an optimal solution of $\mathcal{D}_{m}$. Fix $\varepsilon, \beta \in(0,1)$ such that

$$
\sum_{k=0}^{p-1}\left(\begin{array}{c}
m \\
k
\end{array}\right) \varepsilon^{k}(1-\varepsilon)^{m-k} \leq \beta .
$$

Under Assumption 1, Theorem 1 of [22] ensures that

$$
\begin{aligned}
& \mathbb{P}^{m}\left\{\left(\delta_{1}, \ldots, \delta_{m}\right) \in \Delta^{m}:\right. \\
& \left.\mathbb{P}\left\{\delta=(\bar{n}, \bar{c}, \bar{A}) \in \Delta: \bar{c}^{\top}+\left(\lambda^{\star}\right)^{\top} \bar{A} \geq 0\right\} \geq 1-\varepsilon\right\} \\
& \geq 1-\beta,
\end{aligned}
$$

i.e., with confidence at least $1-\beta$ (measured with respect to $\mathbb{P}^{m}$ ), the optimal solution $\lambda^{\star}$ of $\mathcal{D}_{m}$ remains feasible for a constraint generated by a new extraction $\delta=(\bar{n}, \bar{c}, \bar{A})$ with probability at least $1-\varepsilon$.

\section{B. Effect of a new agent arriving}

Consider a new agent arriving, being characterized by the tuple $\delta=(\bar{n}, \bar{c}, \bar{A})$, thus giving rise to $\mathcal{P}_{m, \delta}$. We will show that, with certain probability, the arrival of the new agent does not alter the optimal solution vector $x^{\star}$ of the $m$ agents, as this was identified by solving $\mathcal{P}_{m}$. Recalling that $x_{\delta}^{\star}$ denotes the optimal solution of $\mathcal{P}_{m, \delta}$, this is summarized in the following theorem.

Theorem 1: Fix any $m \in \mathbb{N}$. Fix $\varepsilon, \beta \in(0,1)$ such that (11) holds. Under Assumption 1,

$$
\begin{aligned}
& \mathbb{P}^{m}\left\{\left(\delta_{1}, \ldots, \delta_{m}\right) \in \Delta^{m}:\right. \\
& \left.\mathbb{P}\left\{\delta=(\bar{n}, \bar{c}, \bar{A}) \in \Delta: x_{\delta}^{\star}=\left(x^{\star}, 0\right)\right\} \geq 1-\varepsilon\right\} \\
& \geq 1-\beta,
\end{aligned}
$$

i.e., with confidence at least $1-\beta, x_{\delta}^{\star}=\left(x^{\star}, 0\right)$ with probability at least $1-\varepsilon$.

Proof: Fix $\delta_{1}, \delta_{2}, \ldots, \delta_{m}$ and consider $\mathcal{P}_{m, \delta}$. Take $x_{\delta}^{v}=$ $\left(x^{\star}, 0\right)$, which is clearly feasible for $\mathcal{P}_{m, \delta}$ and moreover it is a vertex of the polyhedron of feasible solutions for $\mathcal{P}_{m, \delta}$. Since $\bar{x}_{\delta}^{v}=0$ (i.e., the variables corresponding to the new agent are 0 ), the new agent will not contribute to the basic components of $x_{\delta}^{v}$. Thus the decomposition of $\left[c^{\top} \bar{c}^{\top}\right]^{\top}$ and $[A \bar{A}]$ corresponding to the basic and non-basic variables of $x_{\delta}^{v}$ will be $\left[c^{\top} \bar{c}^{\top}\right]_{B}^{\top}=c_{B},[A \bar{A}]_{B}=A_{B}$ and $\left[c^{\top} \bar{c}^{\top}\right]_{N}^{\top}=$ $\left[c_{N}^{\top} \bar{c}^{\top}\right]^{\top},[A \bar{A}]_{N}=\left[A_{N} \bar{A}\right]$, where, we recall, $A_{B}, A_{N}$ and $c_{B}, c_{N}$ are the partition of $A$ and $c$ corresponding to basic and non-basic variables of $x^{\star}$, the optimal solution to $\mathcal{P}_{m}$.

Using Proposition 1 for $\mathcal{P}_{m, \delta}$, which has +1 agents, and given that $c_{N}^{\top}-c_{B}^{\top} A_{B}^{-1} A_{N} \geq 0$ because of Proposition 1 applied to $\mathcal{P}_{m}$, it holds that $x_{\delta}^{v}=\left(x^{\star}, 0\right)$ is optimal for $\mathcal{P}_{m, \delta}$ if and only if

$$
\bar{c}^{\top}-c_{B}^{\top} A_{B}^{-1} \bar{A} \geq 0 .
$$

In turn, since by Proposition $2, \lambda^{\star}=-c_{B}^{\top} A_{B}^{-1}$, (14) is equivalent to

$$
\bar{c}^{\top}+\left(\lambda^{\star}\right)^{\top} \bar{A} \geq 0
$$

Thus, in conclusion, $x_{\delta}^{\star}=\left(x^{\star}, 0\right)$ if and only if (15) holds. Therefore,

$$
\begin{aligned}
& \mathbb{P}\left\{\delta=(\bar{n}, \bar{c}, \bar{A}) \in \Delta: x_{\delta}^{\star}=\left(x^{\star}, 0\right)\right\} \\
& =\mathbb{P}\left\{\delta=(\bar{n}, \bar{c}, \bar{A}) \in \Delta: \bar{c}^{\top}+\left(\lambda^{\star}\right)^{\top} \bar{A} \geq 0\right\} .
\end{aligned}
$$

For any $\varepsilon, \beta \in(0,1)$ such that (11) holds, this latter relation together with (12) gives (13). This concludes the proof.

Theorem 1 serves as a probabilistic perturbation analysis, since it quantifies in a probabilistic sense the effect of the introduction of a new agent (modeled by means of an additional term/perturbation in the coupling constraints) on the objective value of the original multi-agent problem.

An alternative interpretation of Theorem 1 is the following. Employing [26], the inequality in (11) can be made explicit with respect to $m$, i.e.,

$$
m \geq \frac{e}{e-1} \frac{1}{\varepsilon}\left(p-1+\ln \frac{1}{\beta}\right)
$$

where $e$ is the Euler number. As a result, given $\varepsilon, \beta \in(0,1)$, if we have a resource sharing problem with $m$ agents, where $m$ is greater than or equal to the quantity in the right-hand side of (16), then arrival of a new agent will not affect the optimal objective value and the optimal solutions of the other agents with certain probability. The appealing feature of (16) is that it depends logarithmically on the confidence level $\beta$, which can be selected to be very small without an unaffordable increase in the number of agents $m$.

It should be noted that, since the scenario approach theory is applied to the dual problem of $\mathcal{P}_{m}$, the number of agents $m$ plays the role of the number of "scenarios" of the random parameters, while $p$ in (16) is the number of coupling constraints of the primal problem $\mathcal{P}_{m}$ which corresponds to the number of decision variables in $\mathcal{D}_{m}$; these are the number of "support constraints" (notion at the basis of the scenario approach theory) for the dual optimal solution.

\section{Interpretation in electric vehicle charging control}

Consider the electric vehicle charging control problem as presented in [27], [28]; an alternative formulation is considered in [5]. The problem consists of finding an optimal overnight charging schedule for a fleet of $m$ vehicles, whose consumption is denoted by $x_{i}, i=1, \ldots, m$. The overall schedule should be compatible with a network-wide constraint (e.g., maximum power that the network can deliver) thus giving rise to a budget type constraint coupling $x_{i}$, $i=1, \ldots, m$, while each of them has to be non-negative. For a more detailed formulation local limitations for each vehicle can be imposed (e.g., desired final state of charge and maximum charging power); extending our analysis to such cases is currently under investigation (see also Section VI). 
This problem exhibits the structure of $\mathcal{P}_{m}$, thus falling into the proposed theoretical framework. Application of Theorem 1 to this problem provides the means to build an incentive mechanism for plug-in electric vehicles sharing resources such as the maximum power that can be delivered by the network. In particular, our result can serve as a decision support tool to determine how likely it is for a new electric vehicle company penetrating the market to obtain benefit in terms of resource sharing given the existing number of market participants. Note that a new agent in this case does not necessarily ought to be a vehicle, but can be a group of vehicles, e.g., a company, instead.

\section{NUMERICAL SIMULATIONS}

To support the theoretical developments of Section IV with numerical evidence, we consider the optimization problem in (2) for several values of $m$, namely $m=10,11,12, \ldots, 100$. Fixing the value of $m$, the parameters of $\mathcal{P}_{m}$ are set as follows. Each agent $i, i=1, \ldots, m$, has only $n_{i}=1$ decision variable and there are $p=3$ resources with $b=$ $\left[\begin{array}{lll}0.5377 & 1.8339 & -2.2588\end{array}\right]^{\top}$. The scalar cost associated to agent $i$ is extracted at random from a chi-squared distribution with one degree of freedom, i.e., $c_{i} \sim \chi^{2}(1)$, and $A_{i}$ are extracted at random (independently from $c_{i}$ and from each other) from a $p$-variate standard normal distribution, i.e., $A_{i} \sim \mathcal{N}_{p}$. Extractions for different agents are independent and identically distributed in conformance with the developed theory (see discussion above (2)). This set-up is captured by the formalism of Section II defining $\delta_{i}=\left(1, c_{i}, A_{i}\right)$ and $\Delta=\{1\} \times \mathbb{R}_{+} \times \mathbb{R}^{p}$, where $\mathbb{R}_{+}=\{\xi \in \mathbb{R}: \quad \xi \geq 0\}$.

Let $x_{m}^{\star}$ be the optimal solution for $m$ agents. According to Theorem 1,

$$
\mathbb{P}\left\{\delta=(1, \bar{c}, \bar{A}) \in \Delta: x_{m, \delta}^{\star}=\left(x_{m}^{\star}, 0\right)\right\} \geq 1-\varepsilon_{m}
$$

holds true with confidence at least $1-\beta$, where $\bar{c} \sim \chi^{2}(1)$ and $\bar{A} \sim \mathcal{N}_{p}$, independently of $c_{i}$ and $A_{i}, i=1,2, \ldots, m$. In (17), we introduced the subscript $m$ in $\varepsilon_{m}$ to emphasize the fact that the probability level alters for different choices of $m$. For a given $m$ and $\beta, \varepsilon_{m}$ satisfies (11).

To validate (17), we estimate its left-hand side with a sample based counterpart $\hat{\mathbb{P}}_{N_{v}}\left\{x_{m, \delta}^{\star}=\left(x_{m}^{\star}, 0\right)\right\}$ computed based on $N_{v}$ validation samples. For $\alpha, \gamma \in(0,1)$, selecting $N_{v}$ according to

$$
N_{v}=\left\lceil\frac{1}{2 \alpha^{2}} \ln \frac{2}{\gamma}\right\rceil
$$

leads to an empirical estimate $\hat{\mathbb{P}}_{N_{v}}\left\{x_{m, \delta}^{\star}=\left(x_{m}^{\star}, 0\right)\right\}$ which differs at most $\alpha$ from the actual probability that appears in the left-hand side of (17), with confidence at least $1-\gamma$ (see Section 3.1.1 in [29]). For all values of $m$, we set $\beta=\gamma=$ $10^{-6}$ and $\alpha=0.01$, resulting in $N_{v}=72544$.

The above procedure was repeated 1000 times for each value of $m$. In Figure 1 we report the maximum and minimum values of $\hat{\mathbb{P}}_{N_{v}}\left\{x_{m, \delta}^{\star}=\left(x_{m}^{\star}, 0\right)\right\}$ (blue shaded area) and $1-\varepsilon_{m}$ (red line) as a function of $m$. It can be observed that (17) is indeed satisfied in all simulation trials as it was expected since, for each $m$, (17) holds true with very high confidence $1-10^{-6}$. For the sake of completeness, in

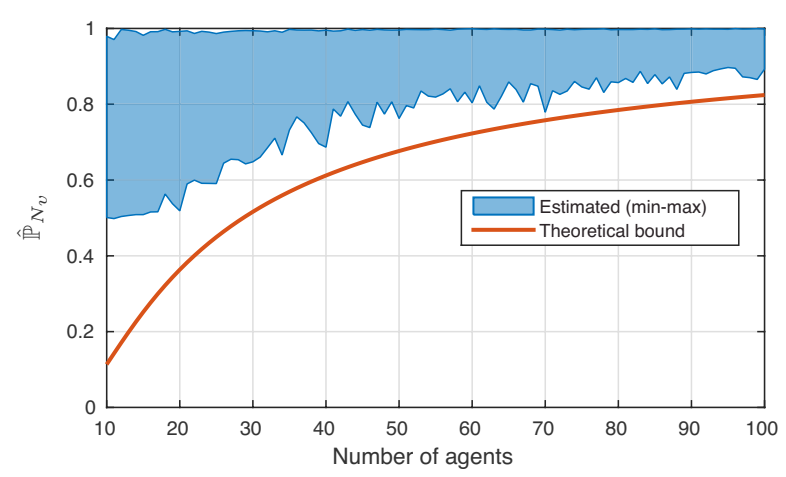

Fig. 1. Maximum and minimum values of the empirical probability $\hat{\mathbb{P}}_{N_{v}}\left\{x_{m, \delta}^{\star}=\left(x_{m}^{\star}, 0\right)\right\}$ over 1000 trials (blue shaded area) and theoretical bound $1-\varepsilon_{m}$ (red line), both as a function of the number of agents $m$.

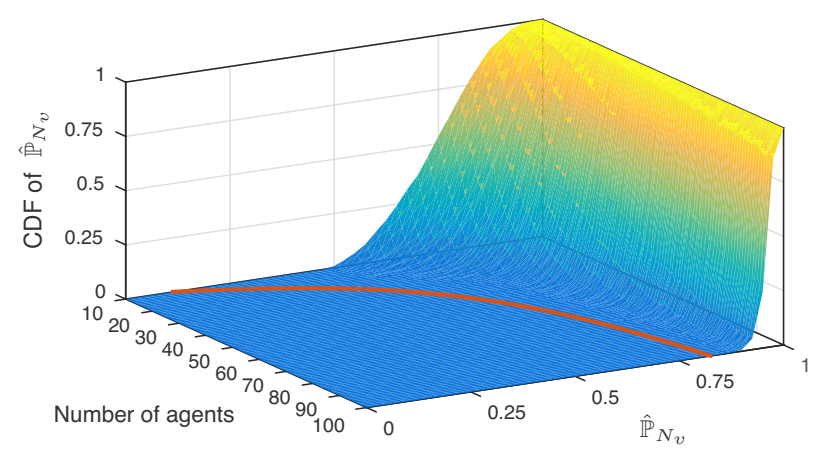

Fig. 2. Empirical Cumulative Distribution Function (CDF) of $\hat{\mathbb{P}}_{N_{v}}\left\{x_{m, \delta}^{\star}=\left(x_{m}^{\star}, 0\right)\right\}$ over 1000 trials (surface) and theoretical bound $1-\varepsilon_{m}$ (red line), both as a function of the number of agents $m$.

Figure 2 we also report the empirical cumulative distribution function of $\hat{\mathbb{P}}_{N_{v}}\left\{x_{m, \delta}^{\star}=\left(x_{m}^{\star}, 0\right)\right\}$ for each value of $m$ (colored surface) together with $1-\varepsilon_{m}$ (red line). As can be seen from the picture, for each $m$, most (more than $1-10^{-6}$ ) of the probability mass of $\hat{\mathbb{P}}_{N_{v}}\left\{x_{m, \delta}^{\star}=\left(x_{m}^{\star}, 0\right)\right\}$ is confined above the $1-\varepsilon_{m}$ threshold as predicted by Theorem 1. Finally, note that $1-\varepsilon_{m}$ approaches 1 (and thus the mass of $\hat{\mathbb{P}}_{N_{v}}\left\{x_{m, \delta}^{\star}=\left(x_{m}^{\star}, 0\right)\right\}$ concentrates around 1) as the number of agents $m$ in the network grows, suggesting that the solution tends to remain unaffected by the arrival of a new agent when the system comprises of a high number of agents.

\section{CONCLUding REMARKS}

In this paper we considered a class of multi-agent resource sharing problem that can be encoded by linear programs. Such problems are often encountered in many application domains that involve different producers/consumers/market participants, like those arising in optimal power flow problems, electric vehicle charging control, etc. The amount of resource to be shared is fixed, while agents are heterogeneous, with each of them contributing to the objective function and the budget type shared resource constraints by a different (linear) term. The latter depends on some uncertain parameters that 
are extracted at random, modelling heterogeneity among agents.

In this context, we studied the probability that the arrival of a new agent leaves the optimal value and the share of the other agents unaffected. As a result, we determined the maximum capacity in terms of the number of agents that the system can accommodate. We followed a probabilistic analysis that involved formulating the dual of the resource sharing linear program, and then showing that this is a random linear program. Using results from the scenario theory for randomized optimization, we constructed a bound on the probability of constraint violation for the dual optimal solution, and showed that this is equivalent with the primal optimal value and resource share remaining unchanged upon the arrival of new agent. We provided also discussion on how this can be thought of as probabilistic sensitivity analysis and offered an interpretation of this setting in an electric vehicle charging control problem.

Current work concentrates towards two directions: i) From a theoretical point of view, we aim at extending the class of resource sharing problems to linear programs with upperbound constraints on the decision variables (here we consider only non-negativity constraints). This will allow us to capture a wider class of problems, e.g., optimal power flow in transmission networks, however, it requires revisiting the theoretical analysis. In particular, in preliminary investigations we shall employ recent results in the scenario approach theory that are based on a "wait-and-judge" paradigm [30], [31], offering a posteriori probabilistic evaluations, in contrast to the a priori result of Theorem 1. ii) From an application point of view, we aim at numerically verifying the efficacy of the presented theoretical results to the electric vehicle charging control problem outlined in Section IVC. Other applications include identifying the probability of price changes in optimal power flow and economic dispatch problems, when a new producer (e.g., intermittent generator) enters the market.

\section{REFERENCES}

[1] J. Warrington, P. Goulart, S. Mariéthoz, and M. Morari, "Policy-based reserves for power systems," IEEE Transactions on Power Systems, vol. 28 , no. 4, pp. 4427 - 4437, 2013.

[2] F. Dörfler, J. Simpson-Porco, and F. Bullo, "Breaking the hierarchy: Distributed control and economic optimality in microgrids," IEEE Trans. on Control of Network Systems, vol. 3, no. 3, 2016.

[3] F. Dörfler and S. Grammatico, "Gather-and-broadcast frequency control in power systems," Automatica, vol. 79, pp. 296-305, 2017.

[4] K. Margellos and S. Oren, "Capacity controlled demand side management: A stochastic pricing analysis," IEEE Transactions on Power Systems, vol. 31, no. 1, pp. 706-717, 2016.

[5] L. Deori, K. Margellos, and M. Prandini, "Price of anarchy in electric vehicle charging control games: When Nash equilibria achieve social welfare," Automatica, under review, pp. 1-10, 2017. [Online]. Available: https://arxiv.org/abs/1612.01342

[6] J. Ghaderi and R. Srikant, "Opinion dynamics in social networks with stubborn agents: Equilibrium and convergence rate," Automatica, vol. 50, pp. 3209-3215, 2014.

[7] S. R. Etesami and T. Başar, "Game-theoretic analysis of the Hegselmann-Krause model for opinion dynamics in finite dimensions," IEEE Trans. on Automatic Control, vol. 60, no. 7, pp. 18861897, 2015.
[8] S. E. Parsegov, A. V. Proskurnikov, R. Tempo, and N. E. Friedkin, "Novel multidimensional models of opinion dynamics in social networks," IEEE Trans. on Automatic Control, vol. 62, no. 5, pp. 22702285, 2017

[9] R. Olfati-Saber and R. Murray, "Consensus problems in networks of agents with switching topology and time-delays," IEEE Trans. on Automatic Control, vol. 49, no. 9, pp. 1520-1533, 2004.

[10] R. Olfati-Saber, "Flocking for multi-agent dynamic systems: Algorithms and theory," IEEE Trans. on Automatic Control, vol. 51, no. 3, pp. 401-420, 2006.

[11] S. Martínez, F. Bullo, J. Cortés, and E. Frazzoli, "On synchronous robotic networks - Part i: Models, tasks, and complexity," IEEE Trans. on Automatic Control, vol. 52, pp. 2199-2213, 2007.

[12] M. Stanković, K. Johansson, and D. Stipanović, "Distributed seeking of Nash equilibria with applications to mobile sensor networks," IEEE Trans. on Automatic Control, vol. 57, no. 4, pp. 904-919, 2012.

[13] D. Bertsekas and J. Tsitsiklis, Parallel and distributed computation: Numerical methods. Athena Scientific (republished in 1997), 1989.

[14] A. Nedić and A. Ozdaglar, "Distributed subgradient methods for multiagent optimization," IEEE Trans. on Automatic Control, vol. 54, no. 1, pp. 48-61, 2009.

[15] A. Nedić, A. Ozdaglar, and P. Parrillo, "Constrained consensus and optimization in multi-agent networks," IEEE Trans. on Automatic Control, vol. 55, no. 4, pp. 922-938, 2010.

[16] M. Zhu and S. Martinez, "On distributed convex optimization under inequality and equality constraints," IEEE Transactions on Automatic Control, vol. 57, no. 1, pp. 151-164, 2012.

[17] K. Margellos, A. Falsone, S. Garatti, and M. Prandini, "Distributed constrained optimization and consensus in uncertain networks via proximal minimization," IEEE Transactions on Automatic Control, to appear, pp. 1-16, 2017, published on-line, doi: 10.1109/TAC.2017.2747505.

[18] F. Parise, M. Colombino, S. Grammatico, and J. Lygeros, "Mean field constrained charging policy for large populations of plug-in electric vehicles," IEEE Conference on Decision and Control, pp. 5101-5106, 2014.

[19] S. Grammatico, F. Parise, M. Colombino, and J. Lygeros, "Decentralized convergence to Nash equilibria in constrained mean field control," IEEE Transactions on Automatic Control, vol. 61, no. 11, pp. 33153329, 2016.

[20] S. Li and W. Zhang, "On Social Optima of Non-Cooperative Mean Field Games," American Control Conference, pp. 3584-3590, 2016.

[21] G. Calafiore and M. Campi, "The scenario approach to robust control design," IEEE Transactions on Automatic Control, vol. 51, no. 5, pp. 742-753, 2006.

[22] M. Campi and S. Garatti, "The exact feasibility of randomized solutions of uncertain convex programs," SIAM Journal on Optimization, vol. 19, no. 3, pp. 1211-1230, 2008.

[23] M. Campi, S. Garatti, and M. Prandini, "The scenario approach for systems and control design," Annual Reviews in Control, vol. 33, no. 2, pp. $149-157,2009$.

[24] D. Bertsimas and J. N. Tsitsiklis, Introduction to linear optimization. Athena Scientific Belmont, MA, 1997, vol. 6.

[25] S. Boyd and L. Vandenberghe, Convex Optimization. Cambridge University Press, 2004.

[26] T. Alamo, R. Tempo, and A. Luque, "On the sample complexity of randomized approaches to the analysis and design under uncertainty," pp. 4671-4676, 2010.

[27] R. Vujanic, P. Mohajerin, P. Goulart, S. Mariéthoz, and M. Morari, "A decomposition method for large scale milps, with performance guarantees and a power system application," Automatica, vol. 67, pp. 144-156, 2016.

[28] A. Falsone, K. Margellos, S. Garatti, and M. Prandini, "Dual decomposition for multi-agent distributed optimization with coupling constraints," Automatica, vol. 84, pp. 149-158, 2017.

[29] M. Vidyasagar, Learning and Generalization with Applications to Neural Networks (2nd eddition). Springer Verlag, 2003.

[30] M. Campi, S. Garatti, and F. Ramponi, "Non-convex scenario optimization with application to system identification," IEEE Conference on Decision and Control, pp. 4023-4028, 2015.

[31] M. Campi and S. Garatti, "Wait-and-judge scenario optimization," Mathematical Programming, pp. 1-35, 2016, published on-line, doi: 10.1007/s10107-016-1056-9. 